\title{
Klinische Aspekte der Biosignalverarbeitung von Atmungsparametern in der Schlafmedizin *
}

\author{
Clinical Views on Biosignal Processing of Ventilation in Sleep Medicine
}

Autoren

Institute
K.-H. Rühle ${ }^{1}$, D. Karweina ${ }^{2}$, U. Domanski ${ }^{1}$, G. Nilius ${ }^{1}$

Klinik für Pneumologie, HELIOS-Klinik Ambrock Hagen. Universität Witten-Herdecke

2 Fachbereich Elektrotechnik und Informationstechnik. Fachhochschule Südwestfalen eingereicht 27.8.2010

akzeptiert 9.9.2010

\section{Bibliografie}

DOI http://dx.doi.org/ 10.1055/s-0030-1255819

Online-Publikation: 18. 10. 2010

Pneumologie 2011; 65:

89-93 @ Georg Thieme

Verlag KG Stuttgart · New York

ISSN 0934-8387

Korrespondenzadresse Prof. Dr. med. Karl-Heinz Rühle HELIOS-Klinik Ambrock Klinik für Pneumologie Ambrockerweg 60 58091 Hagen Klinik-Ambrock.Pneumo@ t-online.de

\section{Zusammenfassung \\ $\nabla$}

Polysomnografisch erfasste Biosignale wie Elektroenzephalogramm (EEG), Elektrookulogramm (EOG) und Elektromyogramm (EMG), Atemfluss, Thorax- und Abdomenbewegung, $\mathrm{O}_{2}$-Sättigung und Puls durchlaufen eine Messkette mit u.a. Verstärkung und Filterung der Messgröße. Durch die Signalbearbeitung wird das ursprüngliche Signal manipuliert, um Informationen zu extrahieren, verschiedene Parameter zu korrelieren und das Signal in verschiedenen Darstellungen zu visualisieren. Die Aufgabe des Klinikers besteht nun darin, aus den ihm zur Verfügung stehenden Informationen bzw. Ergebnissen klinische Rückschlüsse zu ziehen. Dazu sollten die Gerätecharakteristika, speziell die Methodik der Signalbearbeitung durch die Gerätehersteller offengelegt werden und damit überprüfbar sein. In der Übersichtsarbeit wird am Beispiel der $\mathrm{O}_{2}$-Sättigung die Konsequenz unterschiedlicher Bearbeitungskriterien auf den Apnoe/Hypopnoeindex (AHI) dargestellt. Biosignale wie z.B. der Atemfluss werden auch vermehrt zur Steuerung von Therapiegeräten bei automatischen CPAP-Geräten (APAP) eingesetzt. Allerdings sind die implementierten Algorithmen häufig nicht bekannt und aus den polysomnografischen Aufzeichnungen unter APAP-Therapie nicht direkt ableitbar. In Modelluntersuchungen mit einem Flussgenerator können definierte Flussmuster simuliert werden. Mittels BenchTests mit simulierten Apnoen und Wachphasen, die aus der Fluss-Charakteristik diagnostiziert werden, können die Methoden der Signalbearbeitung durch Messung der Druckverläufe überprüft und damit auch für den Kliniker durchschaubar gemacht werden.

\section{Abstract \\ $\nabla$}

Biological signals recorded by polysomnography such as electroencephalograms (EEG), electrooculograms (EOG) und electromyograms (EMG), airflow, thoracic and abdominal motions, $\mathrm{O}_{2}$ saturation and heart frequency pass through a processing chain with amplification and filtering of the measured variables. In the signal processing the original signal is manipulated to extract information in order to correlate various parameters and to visualise the signal in different representations. The task of the clinician is now to take advantage of the information available and to come to clinical conclusions. Therefore the characteristics, especially the methodology of the signal processing in the devices, should be disclosed by the manufacturers and thus be verifiable. In this review for the example of $\mathrm{O}_{2}$ saturation, the consequence of different processing criteria with regard to the apnea/hypopnea index is presented. Biosignals such as the respiratory flow are also increasingly being used to control therapy equipment for automatic CPAP devices (APAP). However, the implemented algorithms are often not known, and cannot directly be derived from the polysomnographic records under APAP therapy. In model experiments with a flow generator, defined flow patterns can be simulated. Using bench tests with simulated apneas and waking periods, diagnosed from the flow characteristics, the methods of signal processing can be verified by measuring the pressure profiles and made more transparent for the clinician.

\footnotetext{
* Erweiterte Fassung eines Vortrags anlässlich der Konferenz „Biosignal 2010“ Advanced Technologies in Intensive Care and Sleep Medicine. Chairs: Kurths J, Penzel T, Malberg H, Wessel N. 14. - 16. Juli 2010 Berlin.
} 


\section{Einleitung}

$\nabla$

Biosignale können dazu verwandt werden, Funktionszustände oder -veränderungen von Organen zu beschreiben oder zu beeinflussen. Im Bereich der Schlafmedizin stehen verschiedene Biosignale, z. B. bioelektrische oder biomechanische Signale, zur Verfügung (siehe auch $\bullet$ Tab. 1).

Eine biologische Messkette besteht aus der Erfassung des primären Messsignals mittels eines Sensors oder Wandlers, der Verarbeitung mit Verstärkung und Filterung, der Analyse und der Darstellung z.B. als numerischer Ausdruck oder Diagramm (siehe - Abb. 1).

Neben EEG, EOG und EMG werden polysomnografisch weitere Biosignale wie Atemfluss, Thorax- und Abdomenbewegung, $\mathrm{O}_{2}$ Sättigung und Puls gemessen, die alle eine Messkette durchlaufen. Signalbearbeitung bedeutet, dass das ursprüngliche Signal manipuliert wird, um Informationen zu extrahieren, verschiedene Parameter zu korrelieren oder das Signal in verschiedenen Modifikationen darzustellen.

Zeitvariate Signale können durch Signalanalyse in ihrer Auswertbarkeit verbessert werden. Die Analyse umfasst die Erkennung und/oder Elimination von biologischen und technischen Störsignalen und dem hochfrequenten Rauschen. Das Rauschen kann durch eine gleitende Mittelwertbildung oder mit Glättungsalgorithmen vermindert werden.

Ein Signalfilter trennt das Signal von anderen parallel übertragenen Signalen. Die Flowkurve enthält z.B. 2 wichtige unterschiedliche Frequenzspektren: Das höherfrequente Signal am Ende einer Inspiration entspricht dem Schnarchen, das getrennt von der Fluss-Zeit-Atmungskurve dargestellt werden kann.
Tab. 1 Einteilung von Biosignalen aufgrund ihrer pysikalischen Eigenschaften. Bioelektrische Signale (EEG,EMG,EOG)

Biomechanische Signale (Atemfluss, Effort, Bewegung, Beschleunigung) Bioakustische Signale (Schnarchen, Lungengeräusche)

Biothermische Signale (Körpertemperatur, Wärme des Atemstroms) Biochemische Signale $\left(\mathrm{PCO}_{2}\right)$

Biooptische Signale (Transmissionsoxymetrie)

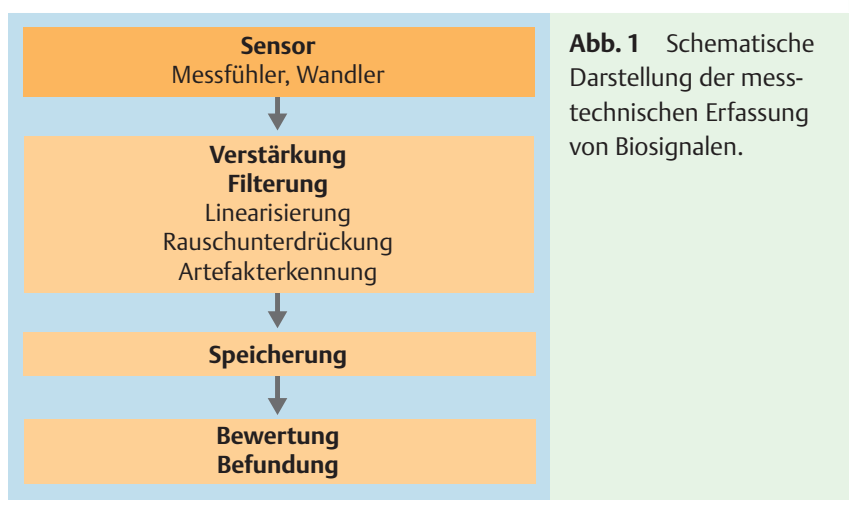

Nur wenn unterschiedliche Charakteristika in der Frequenz der interessierenden Signale oder von Nutz- und Störsignal zu finden sind, ist der Einsatz eines Signalfilters sinnvoll. Häufige Filterarten sind Tiefpass- und Hochpassfilter (siehe Abb. 2).
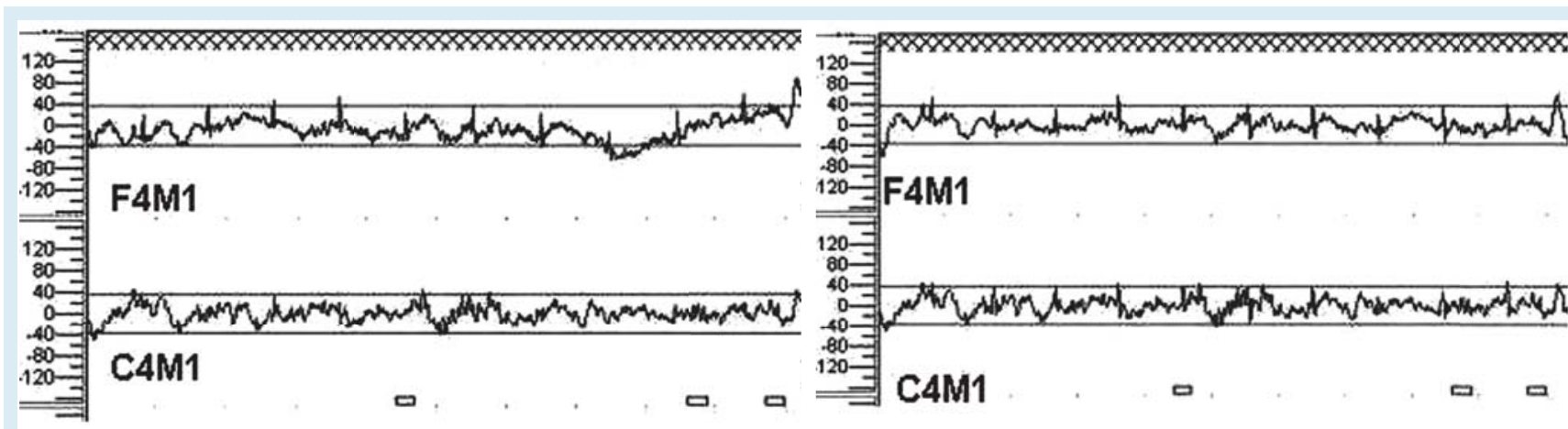

a

b
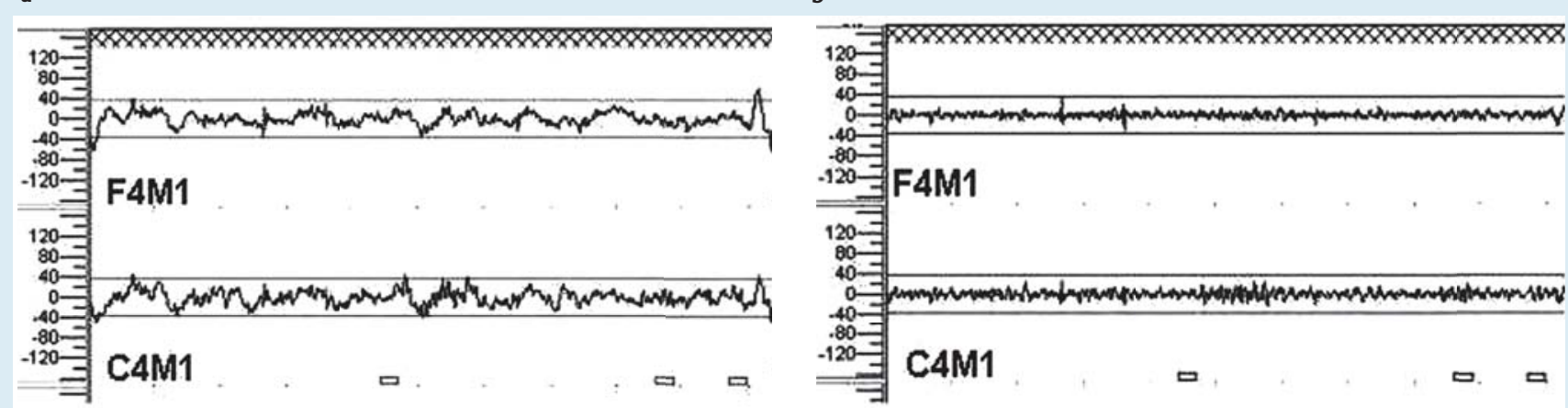

c

d

Abb. 2 Effekt einer Filterung von EEG-Signalen in Schlafstadium N2. Aufzeichnung mit einer Registrierfrequenz von $100 \mathrm{~Hz}$, einer Bandbreite $0,3-45 \mathrm{~Hz}$ bei $3 \mathrm{~dB}$ und Netzfilter in allen 4 Diagrammen. a Ungefilterte Aufzeichnung. Deutliche Schwankungen um Nulllinie mit Einstreuung des EKG. b Hochpassfilter $0,5 \mathrm{~Hz}$, Tiefpassfilter $35 \mathrm{~Hz}$, QRS-Filter ausgeschaltet. Deutlich stabilerer Kurvenverlauf, weiterhin biologische EKG-Artefakte. c Hochpassfilter 0,5 Hz, Tiefpassfilter $35 \mathrm{~Hz}$, QRS-Filter eingeschaltet. Durch den QRS-Filter praktisch vollständige Elimination von EKG-Artefakte. d Hochpassfilter 5,0 Hz, Tiefpassfilter $45 \mathrm{~Hz}$, QRS-Filter eingeschaltet. Durch zu starke Filterung geht die Information des EEG-Nutzsignals verloren. 
Ein Tiefpassfilter lässt dann Signale mit tiefer Frequenz, ein Hochpassfilter nur Frequenzsignale oberhalb einer Grenzfrequenz passieren. Bei der Analyse des EEGs und des EMGs kommt häufig ein $50 \mathrm{~Hz}$-Bandpassfilter zum Einsatz, der das $50 \mathrm{~Hz}$-Signal möglichst stark abschwächt. Der Einfluss biologischer Artefakte im EEG (EOG und EKG) kann durch parallele Registrierung und/ oder entsprechende Signalbearbeitung minimiert werden.

Die Aufgabe des Klinikers besteht nun darin, aus den ihm zur Verfügung stehenden Informationen bzw. Ergebnissen klinische Rückschlüsse zu ziehen.

Dazu ist es erforderlich, nicht nur entsprechende Referenzdaten, sondern auch die Art der Signalbearbeitung zu kennen. Durch die Bearbeitung der Daten kann das Ergebnis abhängig von der Manipulation unterschiedlich ausfallen. Dies hat wiederum Auswirkungen auf die Konsequenzen, die aus dem Befund gezogen werden.

1. Im Folgenden soll anhand der $\mathrm{O}_{2}$-Sättigungsmessung demonstriert werden, in welcher Form sich die Bearbeitung der Daten auf die Bestimmung des Hypopnoe-Index auswirkt.

2. Nicht nur bei diagnostischen Verfahren, sondern auch in der Therapie werden vermehrt Biosignale zur Steuerung bestimmter Abläufe, z.B. bei der automatischen CPAP-Therapie, verarbeitet. Auch hier kennt der Arzt die in den Geräten programmierten Algorithmen häufig nicht. Nur wenn ihm die entsprechenden Regeln und Reaktionsmuster bekannt sind, ist er in der Lage, das Ergebnis zu beurteilen und die richtige Therapieentscheidung zu treffen. Im zweiten Teil dieser Übersicht wird deshalb auf die Gerätesteuerung von automatischen CPAP-Geräten durch Biosignale näher eingegangen.

\section{Diagnostik}

$\nabla$

Um die Auswertung der Sauerstoffsättigung zu vereinfachen, werden die Werte in den verschiedenen Oxymetern über mehrere Sekunden gemittelt. In einer Studie von Davila et al. [1] wurde die Sauerstoffsättigung bei demselben Patienten mit drei Pulsoximetern mit verschiedenen Zeiten der Mittelwertbildung (3 Sekunden, 6 Sekunden und 12 Sekunden) verglichen. Unter der 12 Sekunden Mittelwertbildung wurden geringere Werte für die Sauerstoffdesaturationen gefunden. Dies hatte zur Folge, dass der Desaturationsindex, gemessen bei 30 Patienten um mehr als 30 Ereignisse/Stunde differierte (siehe Tab. 2).

Diese Beobachtung hat Bedeutung bezüglich der Klassifizierung von Hypopnoen nach den Kriterien der American Academy of Sleep Medicine (AASM) [2,3]. Das Scoring einer Hypopnoe erfolgt immer dann, wenn die Flussamplitude um 30\% reduziert ist und die Sauerstoffsättigung um mehr als 4\% absinkt. In den USA und zunehmend auch in Deutschland machen die Versicherungen bzw. Krankenkassen die Bezahlung eines Therapiegerätes von der Höhe des AHI abhängig. Nach den neuen AASM-Regeln wird deshalb für die Aufzeichnung der Sauerstoffsättigung eine maximale Mittelung von drei Sekunden empfohlen.

Während Davila et al. [1] das gleiche Oxymeter mit verschiedenen Mittelungszeiten verwandten, untersuchten Zafar et al. [4] vier verschiedene Oxymeter. Bei einem Gerät kam es im Vergleich zu den anderen Geräten bei 7 von 35 Patienten (d.h. 20\%) mit leicht- bis mittelgradigem AHI-Befund nach Festlegung des Schwellenwertes für den AHI > 15 zu einer Umklassifikation, sodass nach Medicare-Kriterien eine CPAP-Therapie bezahlt werden musste. Da die Mittelwertbildung bei allen Geräten auf den gleichen Wert eingestellt worden war, dürften andere Effekte
Tab. 2 Abhängigkeit der RDE-Frequenz von der zeitlichen Mittelung der tiefsten $\mathrm{O}_{2}$-Sättigung über 3, 6 und 9 Sekunden. $\mathrm{RDE}=$ respiratory disturbance event, definiert durch ein Apnoe/Hypopnoe-Ereignis mit folgender Desaturation um mehr oder weniger als $3 \%$ [1].

\begin{tabular}{|c|c|c|}
\hline Mittelung & RDE $<3 \%$ & RDE $>3 \%$ \\
\hline 3 sek & 13,93 & 73,97 \\
\hline 6 sek & 23,50 & 62,40 \\
\hline 12 sek & 38,40 & 41,93 \\
\hline
\end{tabular}

Tab. 3 Biosignale, die zur Steuerung von Auto-CPAP (APAP) benutzt werden.

Flussamplitude

Flussabflachung (Flattening)

Forcierte Oszillationstechnik (FOT)

Schnarchen

Obstructive pressure peak

Irreguläre Atmung

der Signalverarbeitung wie Rauschunterdrückung oder Filterung die Differenzen verursacht haben.

Für den Kliniker spielt die Biosignalverarbeitung, insbesondere der $\mathrm{O}_{2}$-Sättigungsmessung, eine wichtige Rolle $[5,6]$. In einer Studie an mehr als 6000 Patienten der Sleep Heart Health Study (SHH) wurde die Prävalenz von kardiovaskulären Erkrankungen in Abhängigkeit von verschiedenen Definitionen für Hypopnoen mit $\geq$ oder $<4 \%$ Desaturation oder Arousals untersucht. Es fand sich nur eine Assoziation, wenn die Desaturation $\geq 4 \%$ betrug. Wenn es um Risikoabschätzung geht, ist es deshalb unerlässlich, dass die Ergebnisse der $\mathrm{O}_{2}$-Sättigungsmessung auch bei Messung mit verschiedenen Oxymetern vergleichbar sind oder Korrekturfaktoren zur Verfügung stehen.

Diese Beispiele zeigen, dass zumindest die Kriterien der Signalbearbeitung und der Aufzeichnungsmodus vom Hersteller offengelegt werden sollen. Medizinische Fachgesellschaften sollten bei Empfehlung berücksichtigen, dass verschiedene Auswertungstechnologien im Einsatz sind und dass möglichst genaue Vorschriften hinsichtlich der Grenzkriterien herausgegeben werden. Die Mediziner, welche die Geräte zum Einsatz bringen, sollten sich über die Gerätecharakteristika genau informieren, um die richtigen Schlüsse ziehen zu können. Bei Publikationen zu Schlafstudien sollten speziell in der Methodik die Signalbearbeitungsparameter offengelegt werden. Nur dann ist es möglich, den in der klinischen Routine erhobenen Parametern die entsprechende Bedeutung, die oftmals in epidemiologischen Studien erarbeitet wurden, zuzuordnen.

\section{Therapie}

$\nabla$

Ein weiteres wichtiges Biosignal, u.a. der Atemfluss, wird zur Steuerung von Therapiegeräten, z.B. automatischen CPAP-Geräten (APAP) eingesetzt $(\checkmark$ Tab. 3 ).

Die CPAP-Therapie ist die häufigste Therapiemethode zur Behandlung des obstruktiven Schlafapnoesyndroms. Jedoch klagen Patienten häufig über zu hohen Druck, sodass die Adhärenz darunter leidet. APAP-Geräte passen entsprechend der variablen Atemwegs-Geometrie automatisch den Druck an und senken damit den durchschnittlichen Therapiedruck [7]. Biosignale wie zum Beispiel der Atemfluss können dazu benutzt werden, den jeweils erforderlichen Druck zu steuern und damit die Obstruktion 
zu beseitigen. Unter bestimmten Bedingungen (Obstruktion hauptsächlich im REM-Schlaf, Lageabhängigkeit der oropharyngealen Obstruktion, Therapiedruck über $10 \mathrm{~cm} \mathrm{H}_{2} \mathrm{O}$ ) kann im Vergleich zur konstanten Überdruckbeatmung die Therapie-Akzeptanz durch APAP erhöht werden [8].

Amplitudenveränderungen und Flusskonturen sowie niederfrequente Fluss-Schwankungen (Schnarchen) werden in den einzelnen APAP-Geräten in unterschiedlichen Algorithmen verwendet, um auf die Obstruktion zu schließen. Bei diesen Geräten kommt es besonders darauf an, die Reaktion der Geräte zu kennen, da nur dann die Therapieergebnisse richtig interpretiert werden können.

In Vergleichstests (bench tests) konnte gezeigt werden, dass bedingt durch unterschiedliche Algorithmen verschiedene Druckcharakteristika der APAP-Therapiegeräte resultierten. [9,10]. Dem Kliniker steht häufig nur das Therapiegerät ohne genaue Beschreibung der Algorithmen zur Verfügung. Unklar ist oftmals, mit welcher aktuellen Software das Gerät arbeitet. Wenn wir das Gerät bei Patienten einsetzen, steht uns nur das Therapieergebnis (der Output) anhand der polysomnografischen Parameter zur Verfügung. Es handelt sich demnach um eine Black Box, deren Signalverarbeitung wir nicht genau kennen.

Wie kann der Kliniker dennoch Einblick in die Funktionsweise der Apparaturen bekommen? Durch die Verwendung eines Flussgenerators wird es möglich, definierte Flusssignale in das APAP-Gerät einzuspeisen. Registriert man den resultierenden Fluss und Druck, kann die Reaktion und damit die Arbeitsweise des Gerätes beurteilt werden. Ein großer Vorteil dieses Vorgehens besteht in der Wiederholbarkeit, sodass durch Eingabe des identischen Flussmusters verschiedene Geräte verglichen werden können.

Wir untersuchten z.B. 3 verschiedene APAP-Geräte mittels der Eingabe von repetitiven Apnoen [11]. Bei einem Ausgangsdruck von jeweils 4 mbar erfolgte der Druckanstieg bis 10 mbar nach einer unterschiedlichen Anzahl von Apnoen (1 -6 repetitive Apnoen) und unterschiedlichen Druckstufen.

Außer der Diagnose von Atemstörungen kann allein aus der Atemflusskurve durch Erkennung von irregulärer Atmung auch der Wachzustand erkannt werden. Bei der Beurteilung der Schlafeffizienz allein aufgrund der visuellen Beurteilung der Fluss-Charakteristik im Vergleich zur PSG fanden Guerrero et al. [12] eine Sensitivität von 58,7\%, eine Spezifität von $96,4 \%$ und einen positiven prädiktiven Wert PPV von $81,3 \%$.

Es handelt sich damit um ein Beispiel, mit dem gezeigt werden kann, welche Möglichkeiten in der Signalverarbeitung stecken.

Automatische CPAP-Geräte (APAP) erhöhen, wie schon erwähnt, bei Apnoen und Hypopnoen den Druck, bis die Atmungsstörung beseitigt ist. Bei Normalisierung der Atmung wird der Druck langsam abgesenkt. Im Wachzustand dagegen wäre es wünschenswert, den Druck schnell auf ein geringeres Niveau abzusenken, da dieser vor allem während der Ausatmung als unangenehm empfunden wird. Neuerdings können Wach- von Schlafphasen aus der Atmungsaufzeichnung (Flusskurve) aufgrund irregulärer Frequenz und Amplitude anhand eines mittels neuronalem Netzwerk entwickelten Algorithmus unterschieden werden [13]. Das artifizielle neuronale Netzwerk (ANN) wurde mit den Daten von visuell aus den Flusskurven diagnostizierten Wachphasen von 50 Patienten trainiert. Am Ende des Trainings wurden durch das ANN irreguläre Atemzüge mit einer Sensitivität von 60\% und einer Spezifität von 99,5\% identifiziert, d. h. etwas mehr als die Hälfte aller irregulären Atemzüge wurde mit hoher Spezifität detektiert. In einer Validierungsstudie mit Auf-

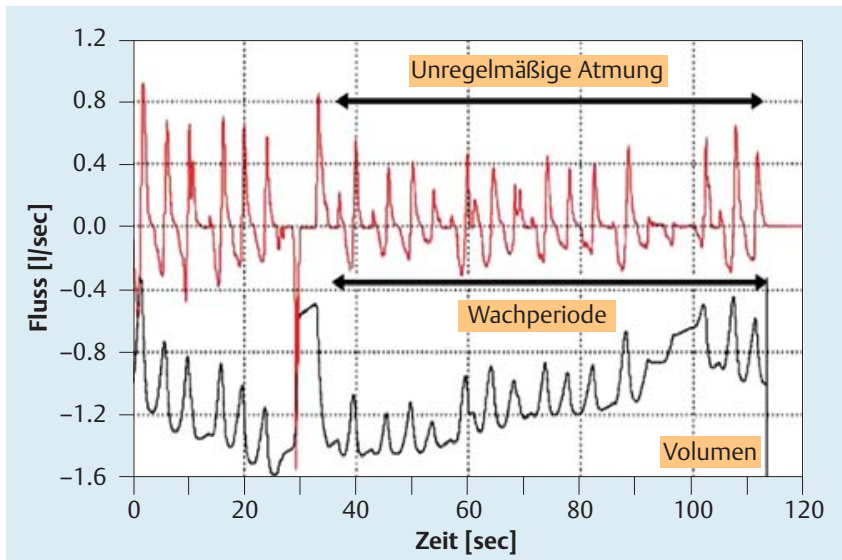

Abb. 3 Beispiel einer Wachperiode mit unregelmäßiger Atmung im Wachzustand. Obere Kurve Fluss in I/sec. Untere Kurve: Registriertes Volumen des Simulators in arbiträren Einheiten. Deutliche Irregularität von Frequenz und Amplitude.

zeichnungen von weiteren 25 Patienten wurde das ANN anhand von EEG definierten Wachphasen überprüft. Der positive prädiktive Wert (PPV = Anzahl richtig positiver/Anzahl richtig positiver + Anzahl falsch positiver) vom Übergang von Schlaf auf Wach oder Arousal lag bei 0,86. Die visuelle Analyse durch einen Somnologen war mit einem PPV von 0,98 dem ANN deutlich überlegen. Dies hing damit zusammen, dass das ANN fälschlicherweise 7\% von irregulärer Atmung dem REM und 7\% dem NREM zuordnete. $70 \%$ von längerem Wachzustand war mit ventilatorischer Irregularität verbunden. Aufgrund dieser Daten war es möglich, ein APAP-Gerät mit einem Algorithmus zu entwickeln, der anhand von Fluss-Kriterien Wachzustände erkennt und demzufolge den Druck schnell absenkt. (Sensawake, Fa. Fisher \& Paykel). Unklar bleibt allerdings, wie zuverlässig der Algorithmus arbeitet. Bei richtiger Reaktion sollte der Druck bei Beginn einer Wachphase absinken.

Wir überprüften deshalb in einer Pilotstudie alle Flussmuster eines Patienten mit obstruktivem Schlafapnoesyndrom (OSAS) während Wachphasen > 2 min. Die Flusskurven im Wachzustand, definiert nach EEG-Kriterien wurden mit einem Flussgenerator simuliert (siehe Abb.3) und die Reaktion des APAP-Gerätes (SleepStyle 200) registriert (siehe $\bullet$ Abb. 4).

Nach Simulation von repetitiven Apnoen wurde der PAP-Wert durch die Steuerlogik des APAP-Gerätes auf den Maximalwert erhöht. Bei fortgesetzter regelmäßiger Atmung sank der PAP geringfügig ab. Dagegen wurde bei mehr als der Hälfte der Wachphasen (57\%) mit beginnender unregelmäßiger Wachatmung der Druck schnell abgesenkt. Relativ regelmäßige Atemmuster im Wachzustand wurden nicht erkannt. Aufgrund dieser ersten Untersuchungen konnte gezeigt werden, dass durch automatische Analyse der Fluss-Charakteristik während einer APAP-Therapie Wacherkennung möglich ist.

Die Erkennung von Wachphasen mittels eines Algorithmus gelingt vor allem bei unregelmäßiger Atmung. Obwohl nicht alle Wachphasen erkannt werden, verspricht die Methode eine verbesserte CPAP-Akzeptanz.

Zusammenfassend besteht das Ziel der Biosignalverarbeitung darin, den Arzt bei der Diagnostik, Befundung und Therapie zu unterstützen. Ohne genaue Kenntnisse der Algorithmen ist eine richtige Beurteilung kaum möglich. Deshalb sollten die Ergebnisse der messtechnischen Erfassung von Biosignalen durch Vergleichsuntersuchungen an Patienten in einen klinischen Kontext 

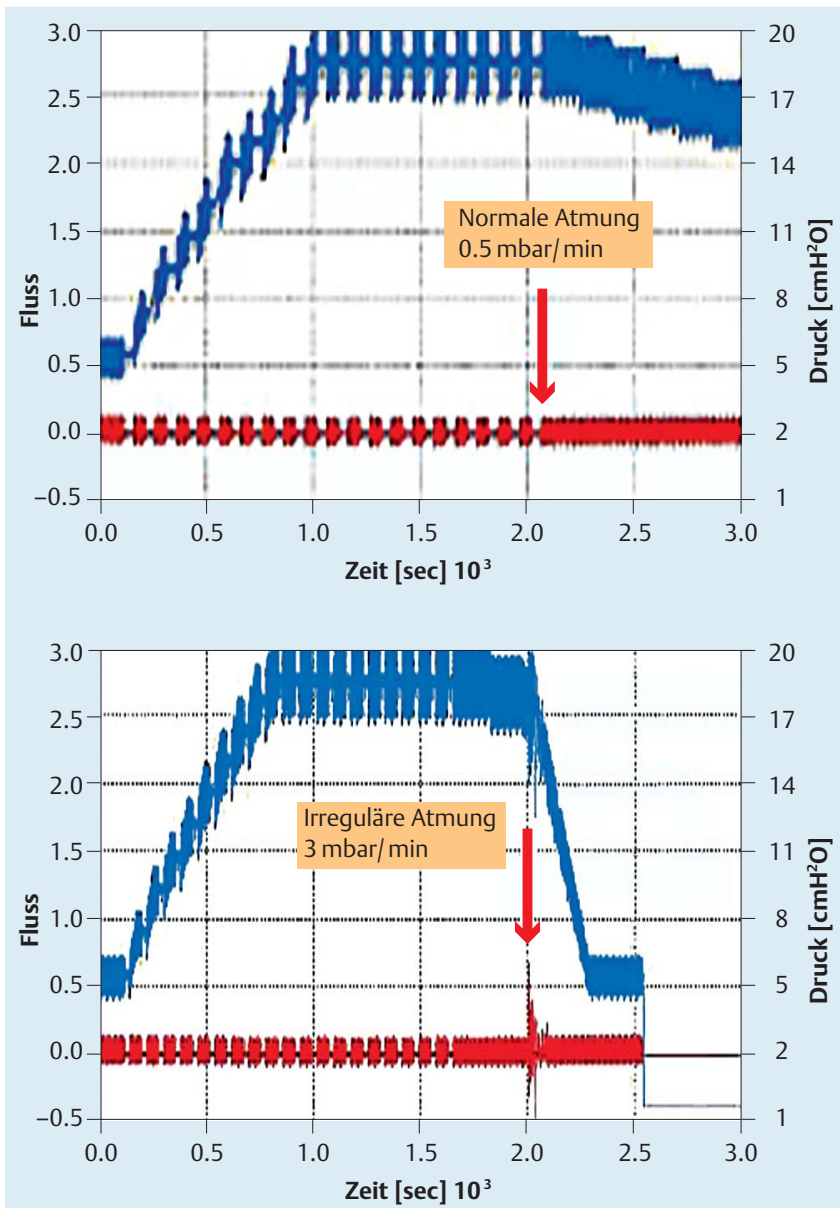

Abb. 4 Testung auf Wachphasenerkennung. Oberes Diagramm: Nach Simulation von repetitiven Apnoen (siehe untere rote Flowkurve) stieg der CPAP-Wert (blaue Kurve) linear bis auf die eingestellte Maximalgrenze von $18,5 \mathrm{mbar}$, um dann konstant zu bleiben. Bei einsetzender regelmäßiger Atmung (Pfeil) sank der CPAP nur um 0,2 mbar/min. Unteres Diagramm: Nach Simulation von repetitiven Apnoen stieg der CPAP-Wert auf die eingestellte Maximalgrenze von 18,5 mbar. Daran anschließend folgte die Simulation der Flusskurve einer irregulären Atmung während einer Wachphase (Pfeil). Richtige Reaktion, da der Druck mit $3 \mathrm{mbar} / \mathrm{min}$ infolge der Detektion einer irregulären Atmung auf 4 mbar erniedrigt wurde.

gestellt werden. Durch Simulation von Biosignalen können komplexe „Black Boxes“ auch für den Kliniker durchsichtig gemacht werden.

\section{Interessenkonflikt}

$\nabla$

K.-H. Rühle und G. Nilius erhielten Studiengelder von Weinmann, Fisher \& Paykel Healthcare, Heinen und Löwenstein, ResMed und TNI. Diese Gelder gingen in wissenschaftliche Projekte der Klinik. D. Karweina und U. Domanski geben an, dass kein Interessenkonflikt besteht.

\section{Literatur}

1 Davila DG, Richards KC, Marshall BL et al. Oximeter's acquisition parameter influences the profile of respiratory disturbances. Sleep 2003; 26: $91-95$

2 Iber C, Ancoli-Israel S, Chesson A, Quan S. For the American Academy of Sleep Medicine. The AASM manual for the scoring of sleep and associated events: rules, terminology and technical specifications. 1st ed. Westchester, IL: American Academy of Sleep Medicine; 2007

3 Ruehland WR, Rochford PD, O'Donoghue FJ et al. The new AASM criteria for scoring hypopneas: impact on the apnea hypopnea index. Sleep 2009; 32: $150-157$

4 Zafar S, Ayappa I, Norman RG et al. Choice of oximeter affects apneahypopnea index. Chest 2005; 127: $80-88$

5 Redline S, Sanders M. A quagmire for clinicians: when technological advances exceed clinical knowledge. Thorax 1999; 54: 474-475

6 Punjabi NM, Newman AB, Young TB et al. Sleep-disordered breathing and cardiovascular disease: an outcome-based definition of hypopneas. Am J Respir Crit Care Med 2008; 177: 1150-1155

7 Randerath WJ, Schraeder O, Galetke W et al. Autoadjusting CPAP therapy based on impedance efficacy, compliance and acceptance. Am J Respir Crit Care Med 2001; 163: 652 - 657

8 Massie CA, McArdle N, Hart RW et al. Comparison between automatic and fixed positive airway pressure therapy in the home. Am J Respir Crit Care Med 2003; 167: 20-23

9 Farré R, Montserrat JM, Rigau J et al. Response of automatic continuous positive airway pressure devices to different sleep breathing patterns: a bench study. Am J Respir Crit Care Med 2002; 166: 469-473

10 Rigau J, Montserrat JM, Wöhrle H et al. Bench model to simulate upper airway obstruction for analyzing automatic continuous positive airway pressure devices. Chest 2006; 130: 350 - 361

11 Rühle KH, Karweina D, Domanski $U$ et al. Regulationsverhalten von Auto-CPAP-Geräten während der Simulation von schlafbezogenen Atemflussmustern. Pneumologie 2009; 63: 390-398

12 Guerrero A, Embid C, Farre R et al. Sleep breathing flow characteristics as a sign for the detection of wakefulness in patients with sleep apnea. Respiration 2009; Epub ahead of print: 3.12.2009

13 Ayappa I, Norman RG, Whiting D et al. Irregular respiration as a marker of wakefulness during titration of CPAP. Sleep 2009; 32(1): 99-104 